\title{
3D Surface Reconstruction of Plant Seeds by Volume Carving
}

Johanna Roussel

j.roussel@fz-juelich.de

Andreas Fischbach

a.fischbach@fz-juelich.de

Siegfried Jahnke

s.jahnke@fz-juelich.de

Hanno Scharr

h.scharr@fz-juelich.de
Institute of Bio- and Geosciences (IBG), IBG-2: Plant Sciences

Forschungszentrum Jülich $\mathrm{GmbH}$

Jülich D-52425, Germany

\begin{abstract}
We describe a method for 3D reconstruction of plant seed surfaces, focusing on small seeds with diameters as small as $200 \mu \mathrm{m}$. The method considers robotized systems allowing single seed handling in order to rotate a single seed in front of a camera. Even though such systems feature high position repeatability, at sub-millimeter object scales, camera pose variations have to be compensated. We do this by robustly estimating the tool center point from each acquired image. 3D reconstruction can then be performed by a simple shape-from-silhouette approach. In experiments we investigate runtimes, the achieved accuracy, and show as a proof of principle that the proposed method is well sufficient for 3D seed phenotyping purposes.
\end{abstract}

\section{Introduction}

Making image analysis methods available for plant phenotyping applications is currently

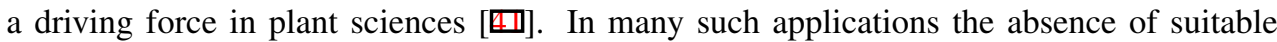
image processing is even a bottleneck [四]. More than 100 specialized methods [ $[\mathbf{Q}$ ] and software packages are available for image-based analysis of different plant parts, e.g. fruit

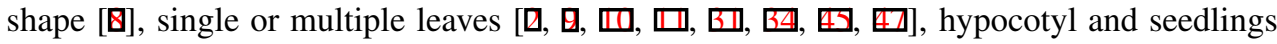

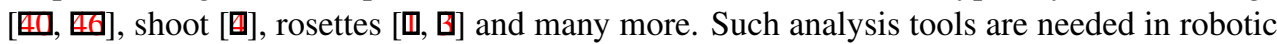

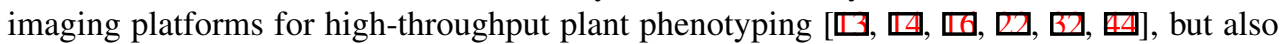
in affordable systems [ [Q8, 四]

Plant seed phenotyping is needed by seed banks for quality management $e . g$. concerning breeding purposes, linking to germination rate or plant growth. For this, 2D scanning is a

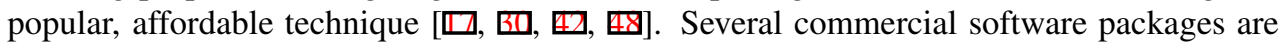
available for seed investigations using flat-bed scanners, e.g. [ㅁ, ष]. It has been applied to different seed types, like Arabidopsis, soybean, barley, or rice. Typically parameters like width, length, or area are calculated from the 2D images, but also more complex shape measures like Fourier descriptors [ $[2, \mathbb{Z}]$. 

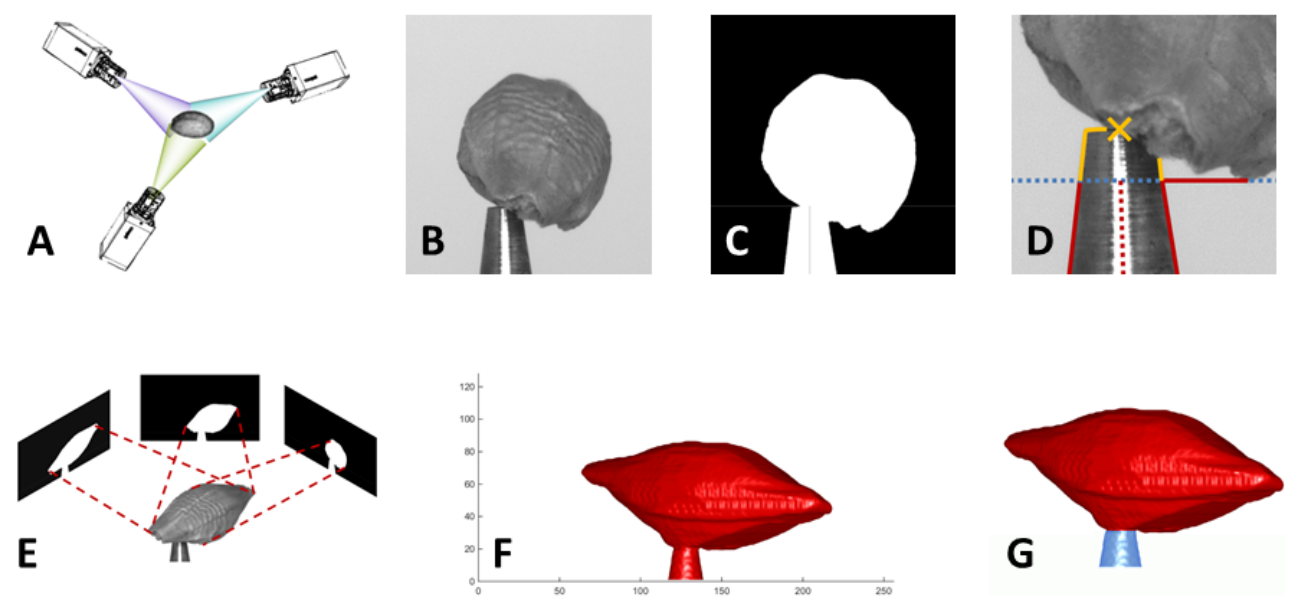

Figure 1: Overview of the reconstruction method. A Image acquisition from multiple viewing angles. B One of the acquired gray value images. C Mask image. D Estimation of tool center point (TCP). E Estimate shape from silhouette by volume carving. F Surface of reconstructed volume. G Tool removed from volume: seed red, tool blue.

3D measurements of seeds have been presented e.g. in [四], where CT scans of Arabidopsis seeds are shown. However, to the best of our knowledge, no affordable 3D imaging technique has been presented so far designed for seed measurements. Correspondence-based techniques $[\mathrm{G}, \mathrm{G}, \mathrm{G}]$ ] reconstructing 3D models from multiple images, or other low-cost techniques like laser scanning or the Kinect can be used for 3D whole plant reconstruction [B]]. However such techniques are not suitable for much smaller objects like seeds of rapeseed plants ( $\sim 2 \mathrm{~mm}$ diameter) or even Arabidopsis seeds ( $\sim 0.2-0.4 \mathrm{~mm}$ length).

Here we investigate volume carving, a well-known shape-from-silhouette technique [ $\square$, $\square, 6]$, for 3D seed shape reconstruction. It is a fast, reliable, and simple but robust method, having been used in plant phenotyping before, e.g. 3D seedling reconstruction [ $[\square]$. Depending on the selected viewpoints it approximates the convex hull of an object or reconstructs even valleys and saddle-points, but cannot reconstruct true concavities. For the seed types investigated here, e.g. barley seeds (see Figure 1), true concavities seem to be of low relevance for volume estimation.

\section{Reconstructing Seed Shape from Silhouettes}

Aiming at relatively simple, mostly convex seed shapes, target voxel resolutions needed to describe such shapes are comparably low - as we will show in the experiments below, see Section 4. Therefore, for this study, it is sufficient to apply one of the most basic volume carving approaches.

We get the intrinsic camera matrix $\mathbf{K}[\mathbf{\square}]$ and the distance between the origin of our working volume and the camera center from calibration ( $\mathrm{cmp}$. Section 3.2). The origin of the working volume is selected to be the tool center point (TCP) of the robot system handling the seeds, i.e. rotating them in front of the camera for imaging (cmp. Section 3.1).

We acquire $N$ images, showing a seed under (equidistantly spaced) rotation angles $\alpha_{i}$ 
where $i \in\{1, \ldots, N\}$, see Figure 1. Rotation is around the vertical axis through the TCP, being parallel to the $y$-axis of the camera. We segment by gray-value thresholding each image into a binary mask $\mathbf{M}_{i}$ being one at the foreground, i.e. seed and tool tip, and zero at background locations. Small objects like noise are removed and small holes (e.g. the reflection of the tool) filled.

For each image and thus segmentation mask we calculate the homogeneous camera projection matrix $\mathbf{P}_{i}$, from the rotation angle $\alpha_{i}$ by

$$
\mathbf{P}_{i}=\mathbf{K}\left(\mathbf{R}_{i} \mid \vec{t}_{i}\right)
$$

where $\mathbf{R}_{i}$ is the rotation matrix corresponding to the given angle $\alpha_{i}$, and translation vector $\vec{t}_{i}$ is calculated using the distance of the world origin to the camera center, also known from calibration (see e.g. [四]). By this, the world coordinate frame rotates with the object, i.e. the seed.

We define an equidistantly spaced, cubic voxel grid around the world origin, being large enough to contain the seed. The thus defined working volume depends on the seed type. For Arabidopsis we use $(1 \mathrm{~mm})^{3}$, for rapeseed $(2.9 \mathrm{~mm})^{3}$, and for barley and maize $(13 \mathrm{~mm})^{3}$.

Each voxel center with homogeneous world coordinates $\vec{X}$ is projected to a point $\vec{x}_{i}$ in each mask $\mathbf{M}_{i}$ by

$$
\vec{x}_{i}=\mathbf{P}_{i} \vec{X}
$$

If $\vec{X}$ is projected to the background region of at least one of the $N$ masks $\mathbf{M}_{i}$, then this voxel does not belong to the foreground object and its value $\mathbf{V}(\vec{X})$ is set to 0 , i.e.

$$
\mathbf{V}(\vec{X})=\prod_{i=1}^{N} \mathbf{M}_{i}\left(\vec{x}_{i}\right)
$$

Thus, if a voxel belongs to the foreground object, its value $\mathbf{V}(\vec{X})$ is set to 1 .

When higher voxel resolution is desired, and thus runtimes increase, parallelization of the carving algorithm [ $[\mathrm{D}]$ is feasible (see Section 4.1). Even higher resolutions become available on current desktop computer hardware, when hierarchically representing the voxel grid, e.g. as an octree [ख].

One of the main drawbacks of this simple carving algorithm is its sensitivity to imprecise external camera calibration. When a mask $\mathbf{M}_{i}$ is misaligned and thus does not well overlap with the 'true' object volume, the non-overlapping parts are deleted from the volume without further testing or corrections. We therefore apply an image-based camera pose calibration step, as described next.

\subsection{Correcting Camera Pose}

Methods not adapting camera pose by estimating extrinsic parameters from the acquired images are known to be particularly sensitive to (extrinsic) calibration errors, thereby requiring precise positioning of the cameras (see e.g. [स] ). For relatively large objects in the multiple centimeter-range, say $20 \mathrm{~cm}$ long and filling most of an image, and typical pixel resolutions, say $2000 \times 2000$, a pixel covers an object area $0.1 \times 0.1 \mathrm{~mm}^{2}$. Position repeatability of industrial-grade robotic systems, typically $\leq 20 \mu \mathrm{m}$ and $\leq 0.05^{\circ}$ [ $\square$ ], is therefore high enough for precise reconstruction. However, for objects being few millimeter in size or even in the sub-millimeter range additional care has to be taken. The mathematical TCP coordinates known to the robot control software may not coincide precisely with the physical 

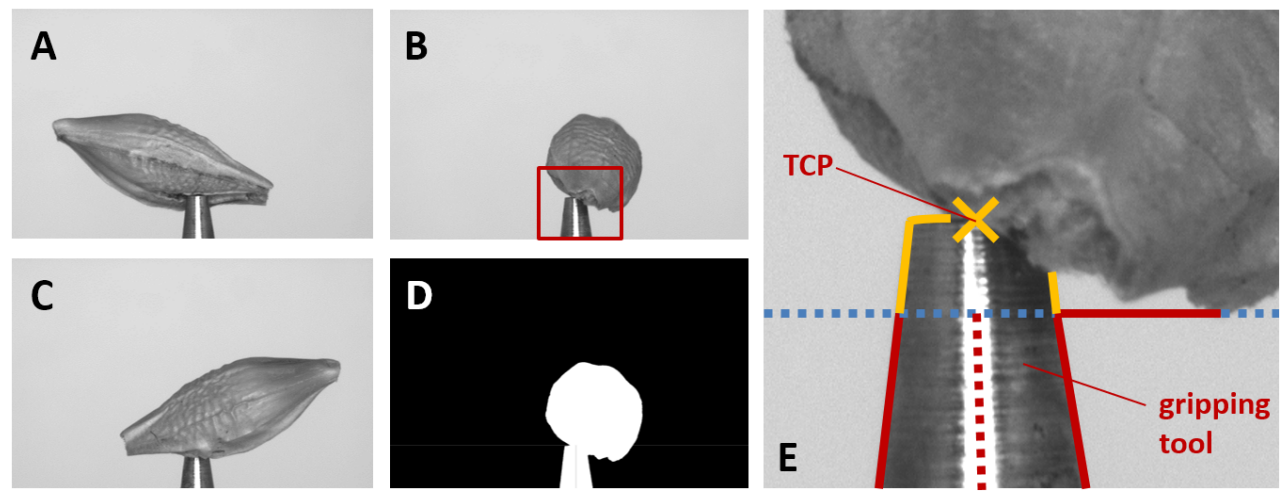

Figure 2: Illustration of the extrinsic camera calibration correction. A-C Images of the same barley seed taken from different angles. D Mask image generated from B. E Steps to find the TCP: (1) find edges of gripping tool (red lines), stop when lines diverge (blue dotted line). (2) $x_{\mathrm{TCP}}$ is average of middle between found edge positions (red dotted line). (3) Trace edges further as long as they come closer to $x_{\mathrm{TCP}}$ (yellow lines). (4) Top most position is $y_{\mathrm{TCP}}$. The found TCP is indicated by a yellow cross.

TCP at the tool tip, due to mechanical calibration inaccuracies, wear and tear, or small deformations of the tool. In our case, instead of being at a fixed location in the camera images, the TCP moves on a more or less reproducible, elliptic trajectory of up to $200 \mu \mathrm{m}$ diameter, varying with room temperature.

Before projecting the voxels to the mask images, we therefore adapt projection matrices $\mathbf{P}_{i}$. To do so, the truncated cone shape of the gripping tool has to be found, see Figure 2. As larger seeds may partly occlude the tool tip, we search for a region of the tool being reliably visible in the images. The tool enters the image vertically from below and becomes smaller in diameter toward the true TCP, being the center point of the very tip of the tool. As we can robustly find the tool's left and right edges, we apply a simple and very fast procedure. We calculate the visible width of the tool line by line starting at the bottom of the image, moving upwards, i.e. in negative $y$-direction. We iterate while the width decreases and is larger than the minimum tool width (being at the tip). The thus reached $y$-coordinate is taken as first estimate of the TCP $y$-coordinate $y_{\mathrm{TCP}}$. A reliable estimate of the TCP's $x$-coordinate $x_{\mathrm{TCP}}$ is established as the mean of all found left and right edge $x$-positions. As the tool tip may be partly occluded by the seed, $y_{\mathrm{TCP}}$ needs refinement. For this the left and right tool edges are independently tracked further until the narrowest point is reached, i.e. the rightmost point of the left edge, and the leftmost point of the right edge. The smallest $y$-value (highest point) of the two points is taken as new $y_{\text {TCP. }}$.

For small seeds like Arabidopsis this procedure works reliably, as the seeds are too small to occlude the whole tool tip in an image. For larger seeds, we use the observation that the TCP's elliptic trajectory results in its $y$-coordinates to describe a sinusoidal curve over the rotation angle. We therefore robustly fit a sin-curve to the $y$-coordinates and correct outliers according to the fit result.

For such small objects, the optical lens setup (cmp. Section 3) features a narrow opening angle (i.e. large zoom), like a microscope at 1-to-1 magnification. This means lines of sight are almost parallel and thus depth effects are negligible. This allows to update $\mathbf{P}_{i}$ with $\vec{x}_{\mathrm{TCP}}$ 
by simply setting the principal point [] to $\vec{x}_{\mathrm{TCP}}$.

When even higher precision of the camera pose would be needed, gradient descent-based algorithms optimizing camera pose may be applied as a refinement step, e.g. [四].

\subsection{Removing the Tool from the Seed}

For small seeds not overlapping with the tool, the TCP lies precisely in the world origin, i.e. the origin of the reconstructed voxel block. Thus voxel above the TCP contain the seed, voxel below (which in that case we do not reconstruct) contain the tool. In cases where seed and tool may overlap (see e.g. Figure 2), the tool tip is also reconstructed. It can be removed from the volume data using its known position, orientation, and physical size by deleting the corresponding voxel volume.

Alternatively, at high voxel resolutions, where the reconstructed volume covered by the tool may be affected by noise, one can estimate the tool position from the reconstruction. Summing up voxel values of horizontal planes in the bottom region of the volume gives reliable estimates of the area of horizontal cuts through the tool. While the areas decrease when summing over higher and higher planes, the planes are deleted from the data. Then, when areas no longer decrease, using these areas, we estimate the $y$-position of the truncated cone using a least squares fit and remove the thus covered volume.

\section{Materials and Methods}

\subsection{Imaging}

Depending on seed size for 3D reconstruction we use two different setups for image acquisition. Both setups consist of an industrial-grade c-mount camera (PointGrey Grasshopper, GRAS-50S5M-C, Mono, 5.0 MP, Sony ICX625 CCD, 2/3", square pixels of size $\mu=3.45 \mu \mathrm{m}$, global shutter, $2448 \times 2048,15$ FPS), $35 \mathrm{~mm}$ high precision lens (Schneider KMP APOXENOPLAN 35/1,9) and a white LED ring with diffusor (CCS LDR2-70-SW2) shown in Figure 3. For small seeds (e.g. Arabidopsis, tobacco, rapeseed) a $36 \mathrm{~mm}$ spacer is mounted between camera and lens. For larger seeds (e.g. barley, maize) only a $15 \mathrm{~mm}$ spacer is needed. Spacer reduce the minimum working distance of the lens $(69.9 \mathrm{~mm}$ for the $36 \mathrm{~mm}$ spacer, $128.0 \mathrm{~mm}$ for the other) and thus are responsible for suitable magnification. This allows to measure seeds in a range between $\approx 0.2 \mathrm{~mm}$ and $12 \mathrm{~mm}$. White paper is used as background.

For image acquisition seeds are picked by a cone-shaped vacuum nozzle and held in front of the camera at optimal working distance using a robotic system to be described elsewhere. The robot rotates the seed in configurable angles and triggers the camera. We use $10^{\circ}$ steps and take 36 images.

\subsection{Camera calibration}

We use the OpenCV implementation [0] of Bouguet's calibration method [0] and an asymmetric $4 \times 11$ dot-pattern target with a total size of $5.8 \times 4.3 \mathrm{~mm}$. It was printed using a professional, high resolution film recorder, as usual office printers even on good paper do not achieve a printing precision suitable for camera calibration at such small spatial scales.

Using this toolbox, estimation of the focal length $f$ is not precise enough for our purposes. We therefore use a ball-bearing ball (steel, precision according to DIN5401 G20) 


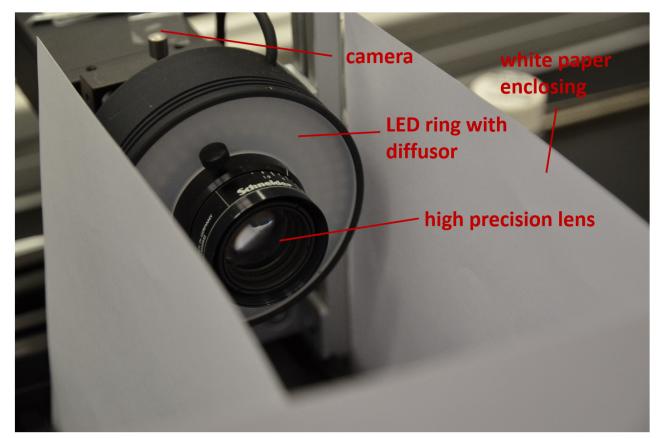

Figure 3: Camera setup for 3D imaging

with $r_{0}=1.50 \mathrm{~mm} \pm 0.25 \mu \mathrm{m}$ radius as calibration object, in order to estimate the working distance $d$ (or equivalently focal length $f$ from working distance $d$ ) of our system precisely. From a mask image of the ball acquired with our system, we estimate its area $A$ in pixel. This allows to estimate its radius $r$ in the image by $r=\mu \sqrt{A / \pi}$, where $\mu$ is the pixel size. From basic geometric reasoning working distance $d$ can be derived as $d=\sqrt{f^{2}+r^{2}} r_{0} / r$.

\subsection{Software Implementation}

The software framework is implemented in $\mathrm{C}++$ on a Windows 7 operating system with Visual Studio 2013. The application programming interface Open Graphics Library (OpenGL [四]) was used for the GPU implementation.

\section{Experiments}

\subsection{Resolution and Runtime}

The complexity of the volume carving algorithm is proportional to the number $N_{V}$ of voxels and number $N$ of images acquired. For our equidistantly spaced cubic $R \times R \times R$ grids the voxel number is $N_{V}=R^{3}$ and thus complexity is $O\left(R^{3} N\right)$. In addition time for loading (or acquiring) the images (with $N_{P}$ pixels) and, for the GPU implementation, transferring the data to and from the graphics card is needed. Complexity of this data transfer and preprocessing of the images is $O\left(N_{P} N\right)$, or $O\left(N_{P} N\right)+O\left(R^{3}\right)$ for the GPU implementation.

Runtimes shown in Figure $4 \mathbf{D}$ have been measured on a PC with Intel Core i5-3470 CPU, 8GB DDR3 RAM and an NVIDIA GeForce GTX 580 GPU with 4047MB GDDR5 RAM (cmp. [Q] for further details). We observe that for low resolutions $R$ of the voxel grid, runtime contributions by the $O\left(N_{P} N\right)$ components dominate, as no dependence on $R$ is visible. For increasing $R$, these parts become negligible. While for the CPU implementation a significant increase of the runtime versus the 2 seconds runtime for smallest voxel resolutions can be noticed at $R=256(4 \mathrm{~s})$, the parallel GPU implementation stays at comparable runtimes even at $R=512$.

When interested in a seed's volume as a trait used for high throughput phenotyping, rather than subtle surface details, voxel resolution can be selected comparably low. In Figure $4 \mathbf{A}-\mathbf{C}$ we show a barley seed and its reconstructions together with its derived volume 


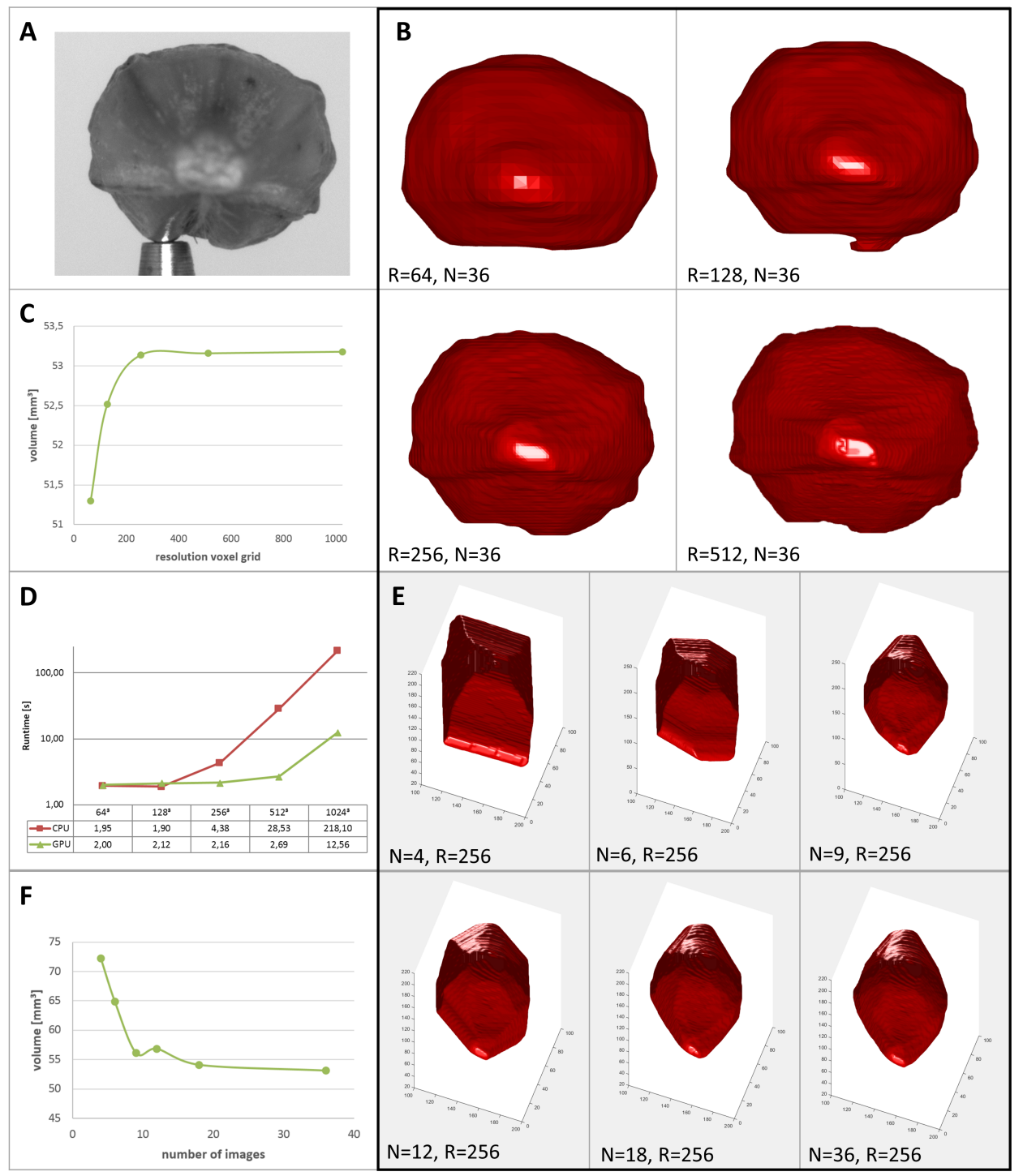

Figure 4: Performance of the proposed method. A Original image of a barley seed. B Reconstructions of the seed at different grid resolutions. C Reconstructed volume versus resolution of the voxel grid. D Runtimes in seconds of serial CPU and parallel GPU implementations (reproduced from [0]). E Reconstructed volume versus number of images used. F Reconstructions of the seed using different numbers of images. 


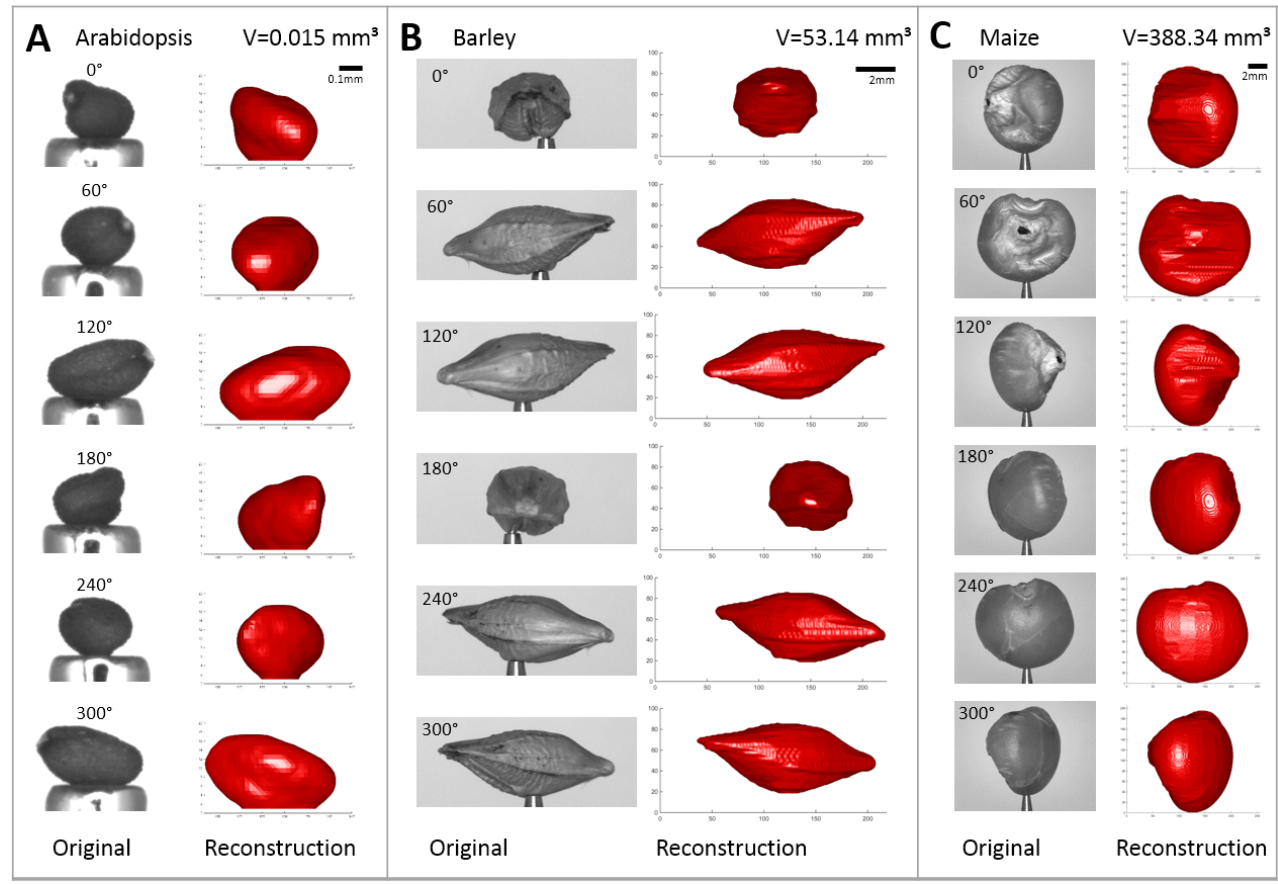

Figure 5: Reconstructed seeds shown from different angles, side-by-side with the original images $(R=256, N=36)$.

for different grid resolutions $R$. We observe that above $R=256$ the estimated volume is approximately constant. Thus, for this phenotyping task, runtime is limited mainly by fileIO, transfer and preprocessing. Sophisticated speed-up mechanisms for the carving provide rather low benefits in this application, as their main potential lies in higher achievable volume resolutions.

Speedup using fewer images may be paid by lower accuracy. We show reconstructions of the same barley seed in Figure $4 \mathbf{E}$ and the corresponding volumes in Figure $4 \mathbf{F}$. Images are selected equidistantly. We observe that reducing image number rapidly reduces reconstruction quality. Interesting to note is that the reconstruction using $N=9$ images is more accurate than with $N=12$ images. This is due to the fact that for $N=12$ the selected angle between images is $\alpha=30^{\circ}$, thus $180^{\circ}$ is a multiple of $\alpha$ (the same is true for $N \in\{4,6,12,18,36\}$ ). However, as the opening angle of our lens is small, complementary information content in masks coming from cameras looking in opposite direction is low. We conclude that for shorter runtimes with comparable or even higher reconstruction accuracy investigating alternative viewing directions is promising.

\subsection{Seed Types and overall Accuracy}

Using the proposed method we reconstructed different seed types, namely Arabidopsis (length, i.e. longest dimension $\lesssim 0.5 \mathrm{~mm})$, rapeseed $(\approx 2 \mathrm{~mm})$, barley $(\approx 8 \mathrm{~mm})$, and maize $(\approx$ $11 \mathrm{~mm}$ ). See Figure 5 to get an impression of the usually achieved reconstruction accuracy.

Absolute accuracy is validated using two different test objects. The first one is the ball- 
bearing ball we used for working distance calibration with $3.00 \mathrm{~mm} \pm 0.5 \mu \mathrm{m}$ diameter, i.e. $0.02 \%$ diameter tolerance, and thus a precisely known volume of $14.137 \mathrm{~mm}^{3} \pm 0.007 \mathrm{~mm}^{3}$, i.e. $0.05 \%$ volume tolerance. Clearly, as we use this object for calibration, scaling of the mask images exactly fit to the respective projection matrices $\mathbf{P}_{i}$. However the overall performance of the system can still be evaluated using this object, as the volume derived has not been used for calibration and still accumulates all errors and imperfections the method has. The second object is an ink cartridge ball with $2.45 \mathrm{~mm} \pm 0.02 \mathrm{~mm}$ diameter, i.e. $0.4 \%$ diameter measurement error, measured with a digital sliding caliper, and thus a volume of $7.70 \mathrm{~mm}^{3} \pm$ $0.094 \mathrm{~mm}^{3}$, i.e. $1.2 \%$ volume error.

Reconstructing the balls using 36 images, a voxel grid resolution of $256^{3}$ and $(12 \mu \mathrm{m})^{3}$ voxel size, yields a volume of $14.15 \mathrm{~mm}^{3}$ and thus a mean radius of $3.0008 \mathrm{~mm}$ for the bearing ball. This is a relative error $w r t$. the specified volume and diameter of $0.08 \%$ (volume) and $0.03 \%$ (diameter). For the ink cartridge ball we measure $7.83 \mathrm{~mm}^{3}$ corresponding to a mean radius of $2.46 \mathrm{~mm}$, being well within the measuring error of our caliper measurement.

By construction, imperfections of the system always result in a loss of volume. Without such errors, we expect to slightly overestimate the volume of the objects we measure, as we carve the volume from the outside - additional images lead to additional loss of volume, cmp. Figure 4E. Slightly overestimating the volumes of the ball targets is therefore a sign for good performance of the calibration and TCP position correction procedures.

We conclude that the overall accuracy of our method, including camera calibration error, mechanical imperfections, TCP finding error, imprecision due to the simple carving approach etc. is high enough to compete with or even beat a precise slide caliper for length measurements. Measurement errors of volume and lengths are well below $0.1 \%$.

\section{Conclusion and Outlook}

Simple volume carving combined with a method for extrinsic camera pose estimation from images is sufficiently accurate for size measurements of even tiny seeds. To optimize our system for runtime and accuracy, we investigated its performance using different parameter settings. Surprisingly, the main performance gain potential does currently not lie in using more sophisticated reconstruction methods allowing for higher achievable voxel resolutions $R, e . g$. achievable by [ $[\mathbb{Z}]$ and necessary for reconstruction of more complex surfaces. Reducing preprocessing and transfer times by reducing image number $N$ and pixel resolution seems more promising.

The method's achievable accuracy has been tested using a spherical object and systematic errors are much lower than we expected. Clearly, as seeds are not well represented by a ball, such accuracy studies give insight in the accuracy potential of the method - if it fails on a ball, it will also fail on more complex shapes.

As seed-shape-specific errors are not well captured by a ball, we plan to build more seedlike objects for ground-truth measurements. Alternative simple volume measurement methods, e.g. Archimedes' principle, are not accurate for such small objects, but high-resolution CT may be an option. Furthermore with a mathematical simulation the over-estimation of the reconstruction can be approximated and used to improve ground truth measurements.

Many factors influence the accuracy, e.g. segmentation errors, small dust particles or camera pose errors. Most critical are inaccuracies of $x_{\mathrm{TCP}}$ and $y_{\mathrm{TCP}}$ in the image, leading to parts of a seed being erroneously carved away. To detect such errors, we plan to implement suitable error estimation, e.g. summing back-projection error. 


\section{References}

[1] E. E. Aksoy, A. Abramov, F. Wörgötter, H. Scharr, A. Fischbach, and B. Dellen. Modeling leaf growth of rosette plants using infrared stereo image sequences. Computers and Electronics in Agriculture, 110:78-90, 2015.

[2] G. Alenya, B. Dellen, and C. Torras. 3D modelling of leaves from color and ToF data for robotized plant measuring. In IEEE International Conference on Robotics and Automation, pages 3408-3414, 2011.

[3] S. Arvidsson, P. Pérez-Rodríguez, and B. Mueller-Roeber. A growth phenotyping pipeline for Arabidopsis thaliana integrating image analysis and rosette area modeling for robust quantification of genotype effects. The New Phytologist, 191(3):895-907, 2011.

[4] M. Augustin, Y. Haxhimusa, W. Busch, and W. G. Kropatsch. Image-based phenotyping of the mature arabidopsis shoot system. In Computer Vision - ECCV 2014 Workshops, volume 8928, pages 231-246. 2015.

[5] J.-Y. Bouguet. Visual Methods for Three-dimensional Modeling. PhD thesis, Pasadena, CA, USA, 1999.

[6] G. Bradski. The OpenCV library. Doctor Dobbs Journal, 25(11):120, 122-125, November 2000.

[7] M. Brenscheidt. Rekonstruktion der visuellen Hülle von Pflanzensamen mithilfe der OpenGL, 2014.

[8] M. T. Brewer, L. Lang, K. Fujimura, N. Dujmovic, S. Gray, and E. van der Knaap. Development of a Controlled Vocabulary and Software Application to Analyze Fruit Shape Variation in Tomato and Other Plant Species. Plant Physiology, 141(1):15-25, May 2006.

[9] M. Bylesjö, V. Segura, R. Y. Soolanayakanahally, A. M. Rae, J. Trygg, P. Gustafsson, S. Jansson, and N. R. Street. Lamina: a tool for rapid quantification of leaf size and shape parameters. BMC plant biology, 8:82, July 2008.

[10] J. De Vylder, D. Ochoa, W. Philips, L. Chaerle, and D. Van Der Straeten. Leaf segmentation and tracking using probabilistic parametric active contours. In Int. Conf. on Computer Vision/Computer Graphics Collaboration Techniques, pages 75-85, 2011.

[11] B. Dellen, H. Scharr, and C. Torras. Growth signatures of rosette plants from time-lapse video. IEEE/ACM Transactions on Computational Biology and Bioinformatics, 2015. In press.

[12] Denso Robotics Europe. Denso main brochure. URL https://www . densorobotics-europe.com/en/robots. EN_Global_EU_042015_V1, accessed June 2015.

[13] N. Fahlgren, M. A. Gehan, and I. Baxter. Lights, camera, action: high-throughput plant phenotyping is ready for a close-up. Current Opinion in Plant Biology, 24(0):93-99, 2015. 
[14] C. Granier, L. Aguirrezabal, K. Chenu, S. J. Cookson, M. Dauzat, P. Hamard, J.-J. Thioux, G. Rolland, S. Bouchier-Combaud, A. Lebaudy, B. Muller, T. Simonneau, and F. Tardieu. PHENOPSIS, an automated platform for reproducible phenotyping of plant responses to soil water deficit in Arabidopsis thaliana permitted the identification of an accession with low sensitivity to soil water deficit. New Phytologist, 169(3):623-635, January 2006.

[15] R. I. Hartley and A. Zisserman. Multiple View Geometry in Computer Vision. Cambridge University Press, ISBN: 0521540518, second edition, 2004.

[16] A. Hartmann, T. Czauderna, R. Hoffmann, N. Stein, and F. Schreiber. HTPheno: An image analysis pipeline for high-throughput plant phenotyping. BMC Bioinformatics, 12(1):148, 2011.

[17] R. P. Herridge, R. C. Day, S. Baldwin, and R. C. Macknight. Rapid analysis of seed size in Arabidopsis for mutant and QTL discovery. Plant Methods, 7(1):3, February 2011.

[18] Next Instruments. Seedcount. URL http://www. nextinstruments.net/ products / seedcount. Accessed June 2015.

[19] Regent Instruments. Winseedle, 2000. URL http://www . regentinstruments.com/assets/winseedle_about.html. Accessed June 2015.

[20] H. Iwata and Y. Ukai. SHAPE: A computer program package for quantitative evaluation of biological shapes based on elliptic fourier descriptors. Journal of Heredity, 93(5): 384-385, 2002.

[21] H. Iwata, K. Ebana, Y. Uga, T. Hayashi, and J.-L. Jannink. Genome-wide association study of grain shape variation among oryza sativa 1 . germplasms based on elliptic fourier analysis. Molecular Breeding, 25(2):203-215, 2010.

[22] M. Jansen, F. Gilmer, B. Biskup, K.A. Nagel, U. Rascher, A. Fischbach, S. Briem, G. Dreissen, S. Tittmann, S. Braun, I. De Jaeger, M. Metzlaff, U. Schurr, H. Scharr, and A. Walter. Simultaneous phenotyping of leaf growth and chlorophyll fluorescence via GROWSCREEN FLUORO allows detection of stress tolerance in Arabidopsis thaliana and other rosette plants. Functional Pant Biology, 36(10/11):902-914, 2009.

[23] M. Klodt and D. Cremers. High-resolution plant shape measurements from multi-view stereo reconstruction. In Lourdes Agapito, Michael M. Bronstein, and Carsten Rother, editors, Computer Vision - ECCV 2014 Workshops, volume 8928 of Lecture Notes in Computer Science, pages 174-184. Springer International Publishing, 2015.

[24] N. J. J. P. Koenderink, M. Wigham, F. Golbach, G. Otten, R. Gerlich, and H. J. van de Zedde. MARVIN: high speed 3d imaging for seedling classification. In Seventh European Conference on Precision Agriculture 2009, pages 279 -286, 2009.

[25] A. Laurentini. The visual hull concept for silhouette-based image understanding. Pattern Analysis and Machine Intelligence, IEEE Transactions on, 16(2):150-162, February 1994. 
[26] G. Lobet, X. Draye, and C. Périlleux. An online database for plant image analysis software tools. Plant methods, 9(1):38, October 2013.

[27] W. N. Martin and J. K. Aggarwal. Volumetric descriptions of objects from multiple views. IEEE Trans. Pattern Anal. Mach. Intell., 5(2):150-158, February 1983.

[28] M. Minervini, M. M. Abdelsamea, and S. A. Tsaftaris. Image-based plant phenotyping with incremental learning and active contours. Ecological Informatics, 23:35-48, September 2014.

[29] M. Minervini, H. Scharr, and S. A. Tsaftaris. Image analysis: The new bottleneck in plant phenotyping [applications corner]. Signal Processing Magazine, IEEE, 32(4): 126-131, July 2015.

[30] C. R. Moore, D. S. Gronwall, N. D. Miller, and E. P. Spalding. Mapping quantitative trait loci affecting arabidopsis thaliana seed morphology features extracted computationally from images. G3 Genes Genomes Genetics, 3(1):109-118, January 2013.

[31] M. Müller-Linow, F. Pinto-Espinosa, H. Scharr, and U. Rascher. The leaf angle distribution of natural plant populations: assessing the canopy with a novel software tool. Plant Methods, 11(1):11, 2015.

[32] K. A. Nagel, A. Putz, F. Gilmer, K. Heinz, A. Fischbach, J. Pfeifer, M. Faget, S. Blossfeld, M. Ernst, C. Dimaki, B. Kastenholz, A.-K. Kleinert, A. Galinski, H. Scharr, F. Fiorani, and U. Schurr. GROWSCREEN-Rhizo is a novel phenotyping robot enabling simultaneous measurements of root and shoot growth for plants grown in soil-filled rhizotrons. Functional Plant Biology, 39:891-904, 2012.

[33] OpenGL.org. Opengl overview, 2015. URL https://www.opengl.org/ about/. accessed June 2015.

[34] J.-M. Pape and C. Klukas. 3-D histogram-based segmentation and leaf detection for rosette plants. In Computer Vision - ECCV 2014 Workshops, volume 8928, pages 6174. 2015.

[35] A. Paproki, X. Sirault, S. Berry, R. Furbank, and J. Fripp. A novel mesh processing based technique for 3d plant analysis. BMC Plant Biology, 12(1):63, 2012.

[36] S. Paulus, J. Behmann, A.-K. Mahlein, L. Plümer, and H. Kuhlmann. Low-cost 3d systems: Suitable tools for plant phenotyping. Sensors, 14(2):3001, 2014.

[37] M. Potmesil. Generating octree models of $3 \mathrm{~d}$ objects from their silhouettes in a sequence of images. Computer Vision, Graphics, and Image Processing, 40(1):1 - 29, 1987.

[38] M. P. Pound, A. P. French, E. H. Murchie, and T. P. Pridmore. Automated recovery of three-dimensional models of plant shoots from multiple color images. Plant Physiology, 166(4):1688-1698, Dec 2014.

[39] L. Quan, P. Tan, G. Zeng, L. Yuan, J. Wang, and S. B. Kang. Image-based plant modeling. ACM Trans. Graph., 25(3):599-604, July 2006. 
[40] L. O. L. A. Silva, M. L. Koga, C. E. Cugnasca, and A. H. R. Costa. Comparative assessment of feature selection and classification techniques for visual inspection of pot plant seedlings. Computers and Electronics in Agriculture, 97:47-55, 2013.

[41] E. P. Spalding and N. D. Miller. Image analysis is driving a renaissance in growth measurement. Current Opinion in Plant Biology, 16(1):100-104, 2013. ISSN 13695266. Growth and development.

[42] T. Tanabata, T. Shibaya, K. Hori, K. Ebana, and M. Yano. Smartgrain: High-throughput phenotyping software for measuring seed shape through image analysis. Plant Physiology, 160(4):1871-1880, 2012.

[43] S.A. Tsaftaris and C. Noutsos. Plant phenotyping with low cost digital cameras and image analytics. In Information Technologies in Environmental Engineering, Environmental Science and Engineering, pages 238-251. Springer Berlin Heidelberg, 2009.

[44] G. van der Heijden, Y. Song, G. Horgan, G. Polder, A. Dieleman, M. Bink, A. Palloix, F. van Eeuwijk, and C. Glasbey. SPICY: towards automated phenotyping of large pepper plants in the greenhouse. Functional Plant Biology, 39(11):870-877, 2012.

[45] M. Wallenberg, M. Felsberg, and P.-E. Forssén. Leaf segmentation using the Kinect. In SSBA'11 Symposium on Image Analysis, 2011.

[46] L. Wang, I. V. Uilecan, A. H. Assadi, C. A. Kozmik, and E. P. Spalding. HYPOTrace: Image Analysis Software for Measuring Hypocotyl Growth and Shape Demonstrated on Arabidopsis Seedlings Undergoing Photomorphogenesis. Plant Physiology, 149(4): 1632-1637, April 2009.

[47] C. Weight, D. Parnham, and R. Waites. LeafAnalyser: a computational method for rapid and large-scale analyses of leaf shape variation. Plant J, 53(3):578-586, 2008.

[48] A. P. Whan, A. B. Smith, C. R. Cavanagh, J.-P. F. Ral, L. M. Shaw, C. A. Howitt, and L. Bischof. GrainScan: a low cost, fast method for grain size and colour measurements. Plant Methods, 10(23):1-10, 2014.

[49] A. J. Yezzi and S. Soatto. Structure from motion for scenes without features. In Computer Vision and Pattern Recognition, 2003. Proceedings. 2003 IEEE Computer Society Conference on, volume 1, pages 525-532, June 2003. 